\title{
Interaction-free Interferometry with Electrons
}

\author{
Amy Turner, Cameron Johnson and Benjamin McMorran
}

University of Oregon, Eugene, Oregon, United States

Atomic reconstructions of biological samples (such as enzymes, ion channels, organelles, and viruses) through transmission electron microscopy (TEM) have enhanced our understanding of fundamental biological functions. [1] The resolution is limited because biological specimens are damaged by the high energy electrons inelastically scattering from the sample: breaking chemical bonds, heating the specimen, and even dislocating atoms. [2] [3] The dose required to provide enough signal to exceed the signal-tonoise ratio induced by shot noise already damages most biological samples. Non-damaging electron imaging requires a dose on the order of $10 \mathrm{e}^{-} / \AA^{2}$. [4] [5] The tension between high resolution and lowdose imaging is prompting exploration into new methods of low-dose TEM imaging, even employing quantum measurement protocols. [3]

Elitzur and Vaidman proposed a nonlocality experiment which detects a bomb without ever interacting with it. [6] Their thought experiment featured an optical Mach-Zehnder interferometer aligned such that there is complete constructive interference at one output of the second beam splitter (the bright port) and destructive interference at the other output (the dark port). If a single photon enters the interferometer, it would always be detected at the bright port regardless of the path it travelled. However, if a highly absorptive sample (like a bomb) was placed in one of the interferometer paths, the probability that the photon is detected at the bright port is no longer unity. Assuming the beam splitters are 50/50, there is a $50 \%$ probability that the photon would be absorbed by the bomb, $25 \%$ probability that the photon is detected at the bright port and $25 \%$ probability that the photon is detected at the dark port. If the photon is detected at the dark port, there is indeed a bomb present and the photon did not trigger the bomb's detonation. Therefore, a detection at the dark port is an interaction-free measurement (IFM). This IFM experiment transpired with photons soon thereafter [7], and has been proposed for electrons [3], as well.

We demonstrate an IFM with electrons using a Mach-Zehnder electron interferometer formed with two nanoscale gratings within a standard TEM, as depicted in Fig. 1(a). The diffraction gratings are milled into $50 \mathrm{~nm}$ thick membranes of amorphous $\mathrm{Si}_{3} \mathrm{~N}_{4}$ using a Focused Ion Beam (FIB) (Fig. 1(b)). [8] These holograms mimic the beam splitters in a light interferometer. The first grating has a blazed profile such that the electron beam is split and focused down to two equal intensity probes at the sample. An aperture is used to block higher-order probes before they reach the sample. A magnetic lens acts as the mirrors, recombining the two interferometer paths at the second grating. The second grating has a binary profile and sorts the beam into light and dark ports. The zeroth order of the second grating is designated as the dark port of interest. Using the projection optics, the interferometer output is focused onto the camera. To align the interferometer, the second grating is moved such that the intensity of the dark port is minimized (Fig. 1(c)), ideally with zero electron counts. When an opaque sample is inserted into one of the interferometer beam paths, electron counts in the dark port increase (Fig. 2(c) and 2(d)). Here we use the sharp edge of a large aperture as the opaque object. This blocks one path of the interferometer, eliminating all interference at the second grating beam splitter (Fig. 2(d)). The measurable rise in dark port current when a sample was inserted into one electron beam is a demonstration of an IFM.

There are achievable and impactful improvements to the experiment that would quickly enhance the results. In this case, the use of gratings that produced two paths with unequal intensity and slight 
misalignments resulted in incomplete destructive interference at the dark port. We plan to eliminate this background signal using improved gratings that produce two beams of equal intensity, zeroing the dark port. This experiment is an important intermediate step on the way to developing quantum electron microscopy. [3]
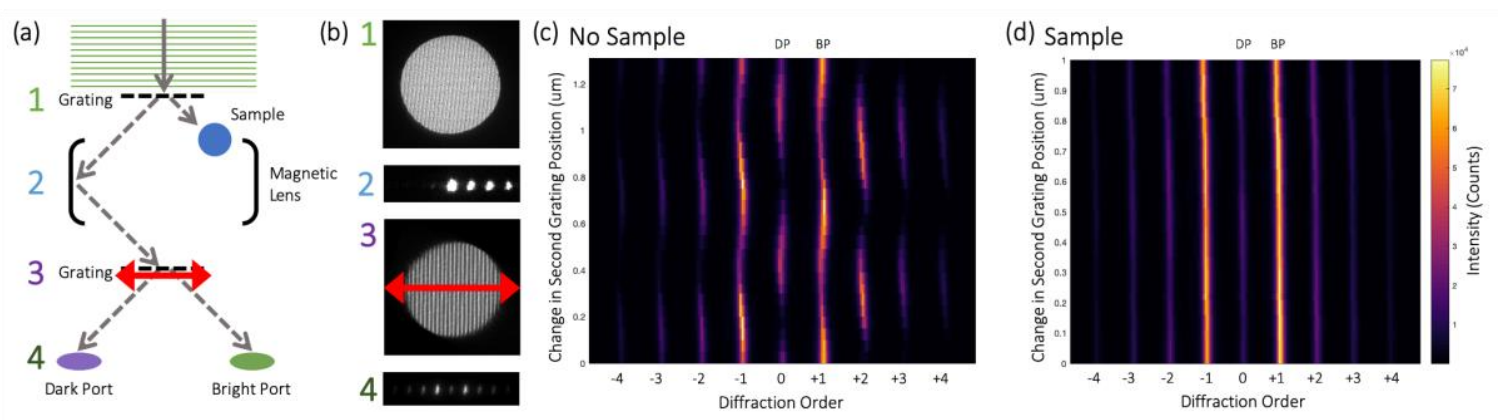

Figure 1. (a) Schematic of electron Mach-Zehnder interferometer in a TEM. (b) Raw images taken in a TEM of the two gratings used (through $10 \mu \mathrm{m}$ diameter aperture) and their diffraction patterns. (c-d) Output from a 2GI as a function of the position of the second grating, with no sample inserted and with a sample blocking one interferometer path.
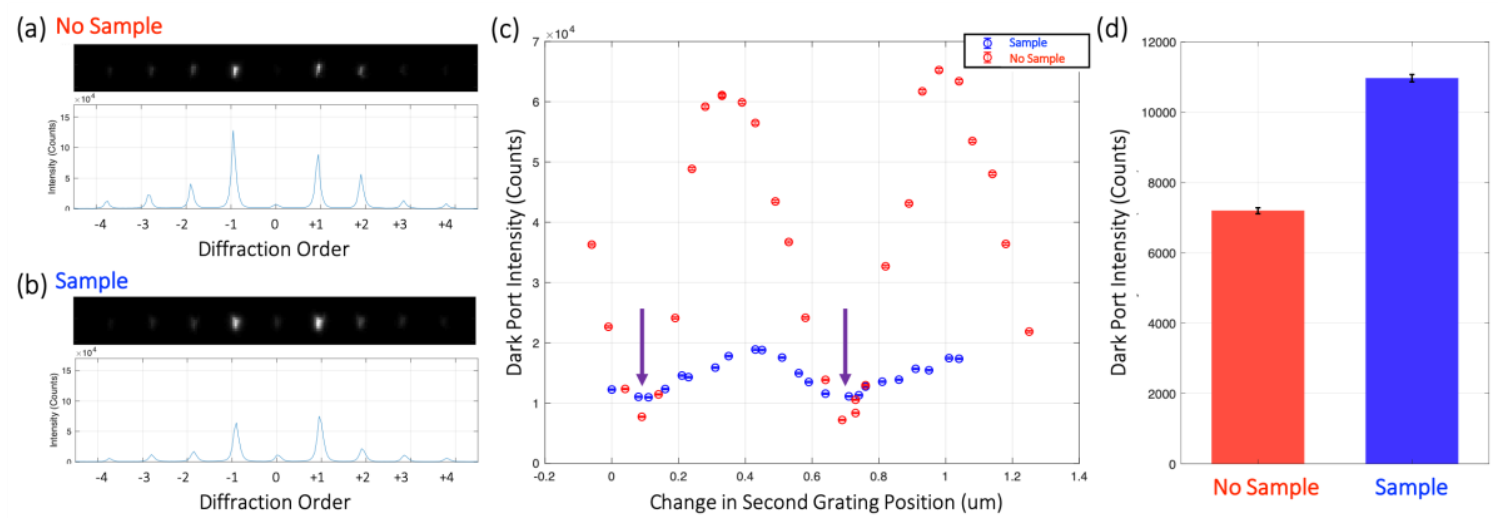

Figure 2. Raw diffraction patterns of an aligned 2GI with (a) no sample inserted and (b) a sample inserted. (c) Intensity of the dark port as a function of the position of the second grating. The purple arrows highlight when the 2GI is aligned and blockage of one path enhances the counts. (d) Dark port electron counts with and without a sample.

\section{References}

[1] R. M. Glaeser, "How Good Can Single-Particle Cryo-EM Become? What Remains Before It Approaches Its Physical Limits?," Annu. Rev. Biophys., vol. 48, no. 1, pp. 45-61, May 2019, doi: 10.1146/annurev-biophys-070317-032828.

[2] R. M. Glaeser and R. J. Hall, "Reaching the Information Limit in Cryo-EM of Biological Macromolecules: Experimental Aspects," Biophys. J., vol. 100, no. 10, pp. 2331-2337, May 2011, doi: 10.1016/j.bpj.2011.04.018.

[3] P. Kruit et al., "Designs for a quantum electron microscope," Ultramicroscopy, vol. 164, pp. 3145, May 2016, doi: 10.1016/j.ultramic.2016.03.004.

[4] R. I. Koning, A. J. Koster, and T. H. Sharp, "Advances in cryo-electron tomography for biology and medicine,” Ann. Anat. - Anat. Anz., vol. 217, pp. 82-96, May 2018, doi: 10.1016/j.aanat.2018.02.004. 
[5] M. Karuppasamy, F. Karimi Nejadasl, M. Vulovic, A. J. Koster, and R. B. G. Ravelli, "Radiation damage in single-particle cryo-electron microscopy: effects of dose and dose rate," J. Synchrotron Radiat., vol. 18, no. 3, pp. 398-412, May 2011, doi: 10.1107/S090904951100820X.

[6] A. C. Elitzur and L. Vaidman, "Quantum mechanical interaction-free measurements," Found. Phys., vol. 23, no. 7, pp. 987-997, Jul. 1993, doi: 10.1007/BF00736012.

[7] P. Kwiat, H. Weinfurter, T. Herzog, A. Zeilinger, and M. A. Kasevich, "Interaction-Free Measurement," Phys. Rev. Lett., vol. 74, no. 24, pp. 4763-4766, Jun. 1995, doi: 10.1103/PhysRevLett.74.4763.

[8] C. W. Johnson, D. H. Bauer, and B. J. McMorran, "Improved control of electron computergenerated holographic grating groove profiles using ion beam gas-assisted etching," Appl. Opt., AO, Aug. 2019. 